\title{
Color Confinement in lattice Landau gauge with unquenched Wilson and KS fermions
}

\section{Hideo Nakajima* and Sadataka Furui ${ }^{\dagger}$}

* Dept. of Infor. Sci., Utsunomiya Univ., Utsunomiya, 320-8585 Japan

E-mail: nakajima@is.utsunomiya-u.ac.jp

$\dagger$ School of Sci.\& Engr., Teikyo Univ., Utsunomiya, 320-8551 Japan

E-mail: furui@umb.teikyo-u.ac.jp

The Kugo-Ojima confinement criterion is verified in the unquenched Landau gauge QCD simulation. The valence quark propagator of the Kogut-Susskind fermion with use of the fermion action including the Naik term and the staple contribution is calculated on MILC Asqtad unquenched gauge configurations, and it shows clear evidence of infrared vanishing of the quark propagator.

XXIIIrd International Symposium on Lattice Field Theory

25-30 July 2005

Trinity College, Dublin, Ireland

${ }^{*}$ Speaker. 


\section{Introduction}

In the lattice Landau gauge QCD simulation, we adopt two types of the gauge field definitions[7]:

$\log U$ type: $U_{x, \mu}=e^{A_{x, \mu}}, A_{x, \mu}^{\dagger}=-A_{x, \mu}$,

$U$ linear type: $A_{x, \mu}=\left.\frac{1}{2}\left(U_{x, \mu}-U_{x, \mu}^{\dagger}\right)\right|_{t r l p .}$,

where ${ }_{t r l}$. implies traceless part. $\left(A_{\mu}(x)=i \sum_{a} A_{\mu}{ }^{a}(x) \frac{\Lambda^{a}}{\sqrt{2}}, \operatorname{tr} \Lambda^{a} \Lambda^{b}=\delta^{a b}\right)$

The corresponding optimizing functions are

$\log U$ type: $F_{U}(g)=\left\|A^{g}\right\|^{2}=\sum_{x, \mu} \operatorname{tr}\left(A_{x, \mu}^{g} A_{x, \mu}^{g}\right)$,

$U$ linear type: $F_{U}(g)=\sum_{x, \mu} \operatorname{tr}\left(2-\left(U_{x, \mu}^{g}+U_{x, \mu}^{g}{ }^{\dagger}\right)\right)$.

Under infinitesimal gauge transformation $g^{-1} \delta g=\varepsilon$, its variation reads for either defintion as

$$
\Delta F_{U}(g)=-2\left\langle\partial A^{g} \mid \varepsilon\right\rangle+\left\langle\varepsilon\left|-\partial D\left(U^{g}\right)\right| \varepsilon\right\rangle+\cdots,
$$

where the covariant derivativative $D_{\mu}(U)$ for two options reads commonly as

$$
D_{\mu}\left(U_{x, \mu}\right) \phi=S\left(U_{x, \mu}\right) \partial_{\mu} \phi+\left[A_{x, \mu}, \bar{\phi}\right]
$$

where $\partial_{\mu} \phi=\phi(x+\mu)-\phi(x)$, and $\bar{\phi}=\frac{\phi(x+\mu)+\phi(x)}{2}$ Stationality of the optimizing function implies Landau gauge, the local minimum implies Gribov Region[2] and the global minimum implies Fundamental modular(FM) region[3].

We performed simulations on quenched configurations tabulated in Table $1[9,10]$ and unquenched configurations of JLQCD[13], CP-PACS[14], Columbia University[15] and MILC[16] tabulated in Table 2[11].

\begin{tabular}{c|c|c|c|c}
$\beta$ & $1 / a(\mathrm{GeV})$ & $L$ & $a L(\mathrm{fm})$ & definition of A \\
\hline 6 & 1.97 & 16 & 1.60 & U-linear/ $\log U$ \\
& & 24 & 2.40 & U-linear/ $\log U$ \\
& & 32 & 3.20 & U-linear/ $\log U$ \\
\hline 6.4 & 3.66 & 32 & 1.72 & U-linear/ $\log U$ \\
& & 48 & 2.59 & U-linear/ $\log U$ \\
& & 56 & 3.02 & U-linear/ $\log U$ \\
\hline 6.45 & 3.87 & 56 & 2.86 & $\log U$
\end{tabular}

Table 1: Configurations used in the quenched QCD simulation

\section{The Kugo-Ojima theory}

The Kugo-Ojima confinement criterion[1] is given by the fact that the parameter $c$ defined as $u^{a b}(0)=-\delta^{a b} c$ in the eq $(2.1)$ becomes 1

$$
\left(\delta_{\mu v}-\frac{q_{\mu} q_{v}}{q^{2}}\right) u^{a b}\left(q^{2}\right)=\frac{1}{V} \sum_{x, y} e^{-i p(x-y)}\left\langle\operatorname{tr}\left(\Lambda^{a \dagger} D_{\mu} \frac{1}{-\partial D}\left[A_{v}, \Lambda^{b}\right]\right)_{x y}\right\rangle .
$$




\begin{tabular}{c|c|c|c|c|c|c|c|c} 
& $\beta$ & $K_{\text {sea }}$ & $a m_{u d}^{V W I} / a m_{s}^{V W I}$ & $N_{f}$ & $1 / a(\mathrm{GeV})$ & $L_{s}$ & $L_{t}$ & $a L_{s}(\mathrm{fm})$ \\
\hline \multirow{2}{*}{ JLQCD } & 5.2 & 0.1340 & 0.134 & 2 & 2.221 & 20 & 48 & 1.78 \\
& 5.2 & 0.1355 & 0.093 & 2 & 2.221 & 20 & 48 & 1.78 \\
\hline CP-PACS & 2.1 & 0.1357 & 0.087 & 2 & 1.834 & 24 & 48 & 2.58 \\
& 2.1 & 0.1382 & 0.020 & 2 & 1.834 & 24 & 48 & 2.58 \\
\hline CU & 5.415 & & 0.025 & 2 & 1.140 & 16 & 32 & 2.77 \\
& 5.7 & & 0.010 & 2 & 2.1 & 16 & 32 & 1.50 \\
\hline MILC $_{c}$ & $6.83\left(\beta_{\text {imp }}\right)$ & & $0.040 / 0.050$ & $2+1$ & 1.64 & 20 & 64 & 2.41 \\
& $6.76\left(\beta_{\text {imp }}\right)$ & & $0.007 / 0.050$ & $2+1$ & 1.64 & 20 & 64 & 2.41 \\
\hline MILC $_{f}$ & $7.11\left(\beta_{\text {imp }}\right)$ & & $0.0124 / 0.031$ & $2+1$ & 2.19 & 28 & 96 & 2.52 \\
& $7.09\left(\beta_{\text {imp }}\right)$ & & $0.0062 / 0.031$ & $2+1$ & 2.19 & 28 & 96 & 2.52 \\
\hline
\end{tabular}

Table 2: Configurations used in the unquenched QCD simulation

The Zwanziger horizon condition[3] coincides with Kugo-Ojima criterion provided the covariant derivative approaches the naive continuum limit, i.e., $e / d=1[8]$. We observe that in the quenched simulation $c$ saturated at about 0.8 , while in the unquenched simulation it is consistent with 1.

\begin{tabular}{c|c|c|c|c|c|c} 
& $K_{\text {sea }}$ or $\beta$ & $c_{x}$ & $c_{t}$ & $c$ & $e / d$ & $h$ \\
\hline JLQCD & $K_{\text {sea }}=0.1340$ & $0.89(9)$ & $0.72(4)$ & $0.85(11)$ & $0.9296(2)$ & $-0.08(11)$ \\
& $K_{\text {sea }}=0.1355$ & $1.01(22)$ & $0.67(5)$ & $0.92(24)$ & $0.9340(1)$ & $-0.01(24)$ \\
\hline CP-PACS & $K_{\text {sea }}=0.1357$ & $0.86(6)$ & $0.76(4)$ & $0.84(7)$ & $0.9388(1)$ & $-0.10(6)$ \\
& $K_{\text {sea }}=0.1382$ & $0.89(9)$ & $0.72(4)$ & $0.85(11)$ & $0.9409(1)$ & $-0.05(9)$ \\
\hline CU & $\beta=5.415$ & $0.84(7)$ & $0.74(4)$ & $0.81(8)$ & $0.9242(3)$ & $-0.11(8)$ \\
& $\beta=5.7$ & $0.95(26)$ & $0.58(6)$ & $0.86(28)$ & $0.9414(2)$ & $-0.08(28)$ \\
\hline MILC $_{c}$ & $\beta=6.76$ & $1.04(11)$ & $0.74(3)$ & $0.97(16)$ & $0.9325(1)$ & $0.03(16)$ \\
& $\beta=6.83$ & $0.99(14)$ & $0.75(3)$ & $0.93(16)$ & $0.9339(1)$ & $-0.00(16)$ \\
\hline MILC $_{f}$ & $\beta=7.09$ & $1.06(13)$ & $0.76(3)$ & $0.99(17)$ & $0.9409(1)$ & $0.04(17)$ \\
& $\beta=7.11$ & $1.05(13)$ & $0.76(3)$ & $0.98(17)$ & $0.9412(1)$ & $0.04(17)$ \\
\hline
\end{tabular}

Table 3: The Kugo-Ojima parameter for the polarization along the spacial directions $c_{x}$ and that along the time direction $c_{t}$ and the average $c$, trace divided by the dimension $e / d$, horizon function deviation $h$ of the unquenched Wilson fermion(JLQCD, CP-PACS), and KS fermion ( MILC $_{c}$, CU,MILC $f$ ). The $\log U$ definition of the gauge field is adopted.

\section{Quark propagator}

In the unquenched lattice simulation with the improved KS fermion action, the MILC collaboration has replaced the link variables by fattening[5]

$$
U_{\mu}(x) \rightarrow c_{1} U_{\mu}(x)+\sum_{v} w_{3} S_{\mu v}^{(3)}(x)+\cdots
$$


where $S_{\mu \nu}^{(3)}$ is the staple contribution

$$
S_{\mu v}^{(3)}(x)=U_{v}(x) U_{\mu}(x+\hat{v}) U_{v}^{\dagger}(x+\hat{\mu})
$$

and added the Naik term which is a product of three link variables along one direction.

The gauge configurations of the MILC collaboration are produced by incorporating, larger staple $S_{\mu v \rho}^{(5)}$ and $S_{\mu v \rho \sigma}^{(7)}$ and the tadpole improvement factor, and the action is called "Asqtad" action.

In the present calculation of valence quark propagator, we do not include all improvements of the "Asqtad" action but incorporate $S^{(3)}$ staple term and the Naik term, i.e. the propagator we measure is the same as that of [5], and we put for Dirac operator $\mathbb{D}+m$ as

$$
\begin{aligned}
\mathbb{D}(U)_{x, y}= & \frac{1}{2} \sum_{\mu=-4}^{4} \eta_{\mu}(x) \operatorname{sign}(\mu)\left[\left(c_{1} U_{\mu}(x)+w_{3} \sum_{v \neq \mu} S_{\mu \nu}^{(3)}(x)\right) \delta_{y, x+\hat{\mu}}\right. \\
& \left.+c_{3} U_{\mu}(x) U_{\mu}(x+\hat{\mu}) U_{\mu}(x+2 \hat{\mu}) \delta_{y, x+3 \hat{\mu}}\right]
\end{aligned}
$$

where $w_{3}=9 / 64, c_{1}=9 / 32$ and $c_{3}=-1 / 24$, and $\eta_{\mu}(x)$ is given as

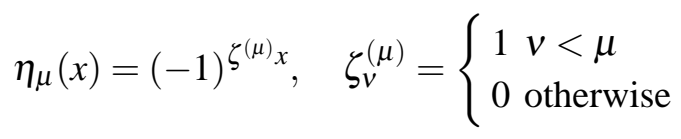

For KS fermion, one defines translation invariant states as

$$
\chi_{p, \alpha}(x)=\frac{1}{\sqrt{V}} e^{i k x}, \quad k_{\mu}=p_{\mu}+\pi \alpha_{\mu}
$$

where $p_{\mu}=2 \pi m_{\mu} / L_{\mu}\left(m_{\mu}=0, \ldots,\left(L_{\mu} / 2\right)-1\right), \alpha_{\mu}=0,1$. Then the Dirac operator in the tree level, $\mathbb{D}(I)+m$, has a proper form in the above basis as

$$
\left\langle\chi_{p^{\prime}, \beta}|\mathbb{D}(I)+m| \chi_{p, \alpha}\right\rangle=\left[i \sum_{\mu}\left(\bar{\gamma}_{\mu}\right)_{\alpha \beta}\left(\frac{9}{8} \sin p_{\mu}-\frac{1}{24} \sin 3 p_{\mu}\right)+m \bar{\delta}_{\alpha \beta}\right] \delta_{p^{\prime} p}
$$

where the Dirac gamma matrices of KS fermions appear as

$$
\left(\bar{\gamma}_{\mu}\right)_{\alpha \beta}=(-1)^{\alpha_{\mu}} \bar{\delta}_{\alpha+\zeta^{(\mu)}, \beta} \quad \text { and } \bar{\delta}_{\alpha \beta}=\prod_{\mu} \delta_{\alpha_{\mu} \beta_{\mu}} .
$$

\section{Calculation of the propagator}

The quark propagator is calculated by statistical average over Landau-gauge-fixed samples as

$$
S_{\alpha \beta}(p)=\left\langle\left\langle\chi_{p, \alpha}\left|\frac{1}{\mathbb{D}(U)+m}\right| \chi_{p, \beta}\right\rangle\right\rangle
$$

The inversion, $\frac{1}{\mathbb{D}(U)+m}$, is performed via conjugate gradient method after preconditioning as follows.

We define the operator $\mathscr{M}=\left(I+\frac{1}{m} \mathbb{D}\right)$ with use of even- odd- sites decomposition

$$
\mathscr{M}=\left(\begin{array}{cc}
I & \frac{1}{m} \mathbb{D}_{o e} \\
\frac{1}{m} \mathbb{D}_{e o} & I
\end{array}\right)=I-L-U
$$


where lower triangle and upper triangle matrices, $L$ and $U$, should be properly understood.

Using the Eisenstat trick[6], we define

$$
\begin{aligned}
\tilde{\mathscr{M}} & =(I-L)^{-1} \mathscr{M}(I-U)^{-1}=(I+L)(I-U-L)(I+U) \\
& =I-L U=\left(\begin{array}{lc}
I & 0 \\
0 I-\left(\frac{1}{m}\right)^{2} & \mathbb{D}_{e o} \mathbb{D}_{o e}
\end{array}\right)
\end{aligned}
$$

where $L^{2}=U^{2}=0$ understood.

We note that $\tilde{\mathscr{M}}$ is hermitian and the conjugate gradient method and/or BiCGstab method are applicable for its inversion. Thus for solution of the equation $m \mathscr{M} \phi=\rho$, we obtain that

$$
\phi=(I+U) \tilde{\mathscr{M}}^{-1}(I+L) \frac{1}{m} \rho
$$

For the right hand side, $\rho$, of the equation $\mathscr{M} \phi=\rho$, we put matrix site field $\rho_{x}=\chi_{p, \beta}(x) I_{3 \times 3}$ where $I_{3 \times 3}$ is the unit color source matrix. Then calculation with use of matrix site field, $\phi,\left\langle\chi_{p, \alpha} \mid \phi\right\rangle$ yields the sample contribution to $S_{\alpha \beta}(p)$ which is color $3 \times 3$ matrix.

Our error estimate of the inversion calculation is

$$
\frac{\|\mathscr{M} \phi-\rho\|}{\|\rho\|}<\text { a few per cent at most }
$$

where the used norm is maximum norm in the space of site, color and flavor, and the accuracy gets $10^{-1}$ higher if $L^{2}$ norm is used.

With use of momentum definition

$$
q_{\mu}=\frac{9}{8} \sin \left(p_{\mu}\right)-\frac{1}{24} \sin \left(3 p_{\mu}\right)=\sin p_{\mu}\left(1+\frac{1}{6} \sin ^{2}\left(p_{\mu}\right)\right),
$$

one obtains that

$$
S_{\alpha \beta}(q)=Z_{2}(q) \frac{-i(\bar{\gamma} q)_{\alpha \beta}+M(q) \bar{\delta}_{\alpha \beta}}{q^{2}+M(q)^{2}}
$$

Since $\operatorname{tr} \bar{\gamma}_{\mu}=0$, trace over color and flavor yields

$$
\operatorname{tr} S(q)=16 N_{c} \frac{Z_{2}(q) M(q)}{q^{2}+M(q)^{2}} \equiv 16 N_{c} \mathscr{B}(q)
$$

On the other hand

$$
\operatorname{tr}(i \bar{\gamma} q S(q))=16 N_{c} q^{2} \frac{Z_{2}(q)}{q^{2}+M(q)^{2}} \equiv 16 N_{c} q^{2} \mathscr{A}(q)
$$

The dynamical mass function of the quark is $M(q)=\frac{\mathscr{B}(q)}{\mathscr{A}(q)}$, and the quark wave function renormalization is $Z_{2}(q)=\frac{\mathscr{A}(q)^{2} q^{2}+\mathscr{B}(q)^{2}}{\mathscr{A}(q)}$. 


\section{Discussion and conclusion}

We found that the Kugo-Ojima parameter is consistent with 1 in the MILC configuration.

The quark field renormalization $Z_{2}$ is found infrared vanishing[12], in contrast to [4]. It implies confinement rather than dynamical chiral symmetry breaking. We observed further, the renormalization of $Z_{2}$ and that of the running coupling are correlated[12]. On the extraction of the continuum limit of the running coupling from compact lattice simulations, there is a warning from the Dyson-Schwinger approach[17].

\section{Acknowledgement}

We acknowledge discussion with Tony Williams, Christian Fischer and Patrick Bowman. This work is supported by the KEK supercomputing project 05-128. H.N. is supported by the JSPS grant in aid of scientific research in priority area No.13135210.

\section{References}

[1] T. Kugo and I. Ojima, Prog. Theor. Phys.(Kyoto) Suppl. 66, 1 (1979).

[2] V.N. Gribov, Nucl. Phys. B 1391(1978).

[3] D. Zwanziger, Nucl. Phys. B 412(1994) 657.

[4] P.O. Bowman et al., Phys. Rev. D71(2005)054507,hep-lat/ 0402032.

[5] K. Orginos and D. Toussaint, Nucl. Phys. B(Proc. Suppl.)73(1999)909.

[6] S. Fischer, A. Frommer, U. Glaässer, Th. Lippert, G. Litzenhöfer and K. Schilling, Comput. Phys. Commun. 98(1996)20.

[7] H. Nakajima and S. Furui, Nucl. Phys. B(Proc. Suppl.)73(1999)865.

[8] H. Nakajima, S. Furui and A.Yamaguchi, Nucl. Phys. B(Proc. Suppl.)94(2001)558.

[9] S. Furui and H. Nakajima, Phys. Rev. D69,074505(2004), hep-lat/0305010.

[10] S. Furui and H. Nakajima, Phys. Rev. D70,094504(2004), hep-lat/0403021.

[11] S. Furui and H. Nakajima, Infrared features of the Kogut-Susskind fermion and the Wilson fermion in Lattice Landau Gauge QCD, hep-lat/0503029.

[12] S. Furui and H. Nakajima, The running coupling in lattice Landau gauge with unquenched Wilson fermion and KS fermion, this proceedings(PoS(LAT2005)291).

[13] S.Aoki et al., (JLQCD collaboration), Phys. Rev. D65,094507(2002); ibid Phys. Rev. D68,054502(2003).

[14] A. Ali Khan et al., (CP-PACS collaboration), Phys. Rev. D65,054505(2002); S. Aoki et al.,(CP-PACS collaboration), Phys. Rev. D60,114508(1999).

[15] F.R. Brown et al.,Phys. Rev. Lett.67,1062(1991).

[16] C.W. Bernard et al., Phys. Rev. D64,054506(2001), hep-lat/0104002

[17] C.S. Fischer, B.Gruter and R. Alkofer, Solving coupled Dyson-Schwinger equations on a compact manifold, hep-ph/0506053. 